\title{
A New One-Sample Log-Rank Test
}

\section{Jianrong $\mathbf{W u}^{*}$}

Department of Biostatistics, St. Jude Children's Research Hospital, 262 Danny Thomas Place, Memphis, TN 38105, USA

\begin{abstract}
The one-sample log-rank test has been frequently used by epidemiologists to compare the survival of a sample to that of a demographically matched standard population. Recently, several researchers have shown that the one-sample log-rank test is conservative. In this article, a modified one-sample log-rank test is proposed and a sample size formula is derived based on its exact variance. Simulation results showed that the proposed test preserves the type I error well and is more efficient than the original one-sample log-rank test.
\end{abstract}

Keywords: Epidemiology; One-sample log-rank test; Time-to-event; Sample size; Standard population

\section{Introduction}

Two-sample log-rank tests are frequently used to design and make inferences for randomized phase III survival trials with two treatment arms. The primary aim of such a study is to compare the survival distributions between two treatment groups. In some cases, it is also interested in comparing the survival distribution of a single sample to that of a standard population. Such comparison arises naturally in epidemiologic studies and clinical trials. For example, in an epidemiologic study, in which the survival data of patients with a life-threatening disease have been prospectively collected, it may be of interest to know if the study sample experiences better survival than the demographically matched standard population. It is not appropriate to use the two-sample log-rank test to make this comparison because the variance could be overestimated; thus, the p-value from the twosample log-rank test is invalid. However, an analog test statistic called the one-sample log-rank test [1] can be used for such study design and comparison.

There is relatively little literature available to design and make inferences for comparing the survival of a sample to a standard population. The one- sample log-rank test was first introduced by Breslow [2]. Its asymptotic property has been studied by Hyde [3], Anderson et al. [4], and Gill and Ware [5], and applications can be found in Finkelstein et al. [1], Berry [6], Woolson [7], and Anderson et al. [4]. Study designs using the one-sample log-rank test were considered by Finkelstein et al. [1]. Kwak and Jung [8], Jung [9], and Sun et al. [10] applied it to single-arm phase II clinical trial designs.

If a study is planned to determine whether the survival of the new study participants better than that of a standard population, then the study must be carefully designed to ensure sufficient power to detect a specific difference of the survival distributions. For the study design, a sample size formula of the one-sample log-rank test is given by Finkelstein et al. [1]. Kwak and Jung [8] proposed another sample size formula for single-arm phase II clinical trial design using the one-sample log-rank test. $\mathrm{Wu}$ [11] recently derived a new sample size formula based on its exact variance. However, simulation results done by Kwak and Jung [8], Sun et al. [10] and $\mathrm{Wu}$ [11] have shown that the one-sample log-rank test is conservative, even when the sample size is relatively large. Thus, it is necessary to develop a new test statistic that preserves the type I error rate and keeps the power as high as possible. Sun et al. [10] derived two corrections of the one-sample log-rank test statistics based on its Edgeworth expansion. However, a major drawback of their corrected tests is that they are more complicated test statistics involving higher-order moment estimations, which makes it difficult to derive their distributions under the alternative. Thus, they can't be used for the study design.

Here we propose a new and simple one-sample log-rank test to correct the conservativeness of the original one-sample log-rank test. A sample size formula is also derived for the new test for the purpose of the study design. The rest of the article is organized as follows. In Section 2, a new one-sample log-rank test is proposed. A sample size formula is derived in Section 3. In Section 4, simulation studies are conducted to compare the empirical type I error and power among four test statistics. An example is given in Section 5. Concluding remarks are given in Section 6.

\section{One-Sample Log-Rank Tests}

The one-sample log-rank test was first introduced by Breslow [2], and it has been used frequently by epidemiologists [3]. To introduce the one-sample log- rank test, let $\Lambda_{0}(x)$ and $S_{0}(x)$ be the known cumulative hazard and survival functions for the standard population, and let $\Lambda(x)$ and $S(x)$ be the unknown cumulative hazard and survival functions for the new study. Then the study may consider the following hypothesis of interest:

$$
H_{0}: S(x) \leq S_{0}(x) \text { vs. } S(x)>S_{0}(x),
$$

or an equivalent to the hypothesis, in terms of cumulative hazard function

$$
H_{0}: \Lambda(x) \leq \Lambda_{0}(x) \text { vs. } \Lambda(x)>\Lambda_{0}(x) .
$$

Suppose during the accrual phase of the trial $n$ subjects are enrolled in the study. Let $T_{i}$ and $C_{i}$ denote, respectively, the failure time and censoring time of the $i^{\text {th }}$ subject. We assume that the failure time $T_{i}$ and censoring time $C_{i}$ are independent and $\left\{T_{i}, C_{i}, i=1, \ldots, n\right\}$ are independent and identically distributed. Then the observed failure time and failure indicator are $X_{i}=T_{i} \Lambda C_{i}$ and $\Delta_{i}=I\left(T_{i} \leq C_{i}\right)$, respectively, for $i^{\text {th }}$ subject. On the basis of the observed data $\left\{X_{i}, \Delta_{i}, i=1, \cdots, n\right\}$, we define

*Corresponding author: Jianrong Wu, Department of Biostatistics, St. Jude Children's Research Hospital, 262 Danny Thomas Place, Memphis, TN 38105 USA, Tel: 901-595-2850; Fax: 901-595-8843; E-mail: jianrong.wu@stjude.org

Received July 30, 2014; Accepted August 15, 2014; Published August 20, 2014

Citation: Wu J (2014) A New One-Sample Log-Rank Test. J Biomet Biostat 5: 210. doi:10.4172/2155-6180.1000210

Copyright: @ 2014 Wu J. This is an open-access article distributed under the terms of the Creative Commons Attribution License, which permits unrestricted use, distribution, and reproduction in any medium, provided the original author and source are credited. 
$O=\sum_{i=1}^{n} \Delta_{i}$ as the observed number of events, and $E=\sum_{i=1}^{n} \Lambda_{0}\left(X_{i}\right)$ as the expected number of events (asymptotically), then the one-sample logrank test is defined by

$$
L_{1}=\frac{O-E}{\sqrt{E}} .
$$

To study the asymptotic distribution of the one-sample log-rank test statistic, we formulate it using counting-process notations [12].

Specifically, let $N_{i}(x)=\Delta_{i} I\left\{X_{i} \leq x\right\}$ and $Y_{i}(x)=I\left\{X_{i} \geq x\right\}$ be the failure and at-risk processes, respectively, then

$$
O=\sum_{i=1}^{n} \int_{0}^{\infty} d N_{i}(x), E=\sum_{i=1}^{n} \int_{0}^{\infty} Y_{i}(x) d \Lambda_{0}(x) .
$$

Thus, the counting-process formulation of the one-sample logrank test is given by

$$
L_{1}=W / \hat{\sigma}
$$

where

$$
W=n^{-1 / 2} \sum_{i=1}^{n} \int_{0}^{\infty}\left\{d N_{i}(x)-Y_{i}(x) d \Lambda_{0}(x)\right\},
$$

and

$$
\hat{\sigma}^{2}=n^{-1} \sum_{i=1}^{n} \int_{0}^{\infty} Y_{i}(x) d \Lambda_{0}(x)
$$

Under the null hypothesis $H_{0}: n^{-1} \sum_{i=1}^{n} Y_{i}(x) \rightarrow G(x) S_{0}(x)$, where $G(x)$ is the survival distribution of censoring time $C$. Thus, $\hat{\sigma}^{2}$ converges to $v^{2}=\int_{0}^{\infty} G(x) S_{0}(x) d \Lambda_{0}(x)$, which is the exact variance of $W$ under the null hypothesis. As showed in the Appendix, the exact mean of $W$ under the null is $E_{H_{0}}(W)=0$. Therefore, by counting process central limit theorem [12], under the null hypothesis, $L_{1}$ is asymptotically standard normal distribution. Hence, we reject the null hypothesis $H_{0}$ with one-sided type I error $\alpha$ if $L_{1}=W / \hat{\sigma}<-z_{1-\alpha}$, where $z_{1-\alpha}$ is the $100(1-\alpha)$ percentile of the standard normal distribution.

Simulation results showed, however, that the one-sample log-rank test $L_{1}$ is conservative, even when the sample size is relatively large [811]. For example, the empirical type I error of $L_{1}$ could be as low as 0.036 for a one-sided type I error rate of 0.05 (Table 1 ). To preserve the type I error, Sun et al. [10] derived two corrections based on Edgeworth expansion which are given below. Let $\hat{\gamma}_{0}=n^{-1} \sum_{i=1}^{n} \Lambda_{0}\left(X_{i}\right)$, $\hat{\gamma}_{1}=(2 n)^{-1} \sum_{i=1}^{n} \Lambda_{0}^{2}\left(X_{i}\right), \hat{k}_{11}=\hat{\gamma}_{1} \hat{\gamma}_{0}^{-3 / 2}$, and $\hat{k}_{12}=\hat{\gamma}_{0}^{-1 / 2}$. Two corrected one-sample log-rank tests are given by

$$
\begin{aligned}
& L_{2}=K_{n}-\frac{1}{\sqrt{n}}\left\{\frac{1}{2} \hat{k}_{11}+\frac{1}{6} \hat{k}_{12}\left(K_{n}^{2}-1\right)\right\} \\
& \text { and } \\
& L_{2}=\frac{1}{\hat{\xi}}\left\{\exp \left(\hat{\xi} K_{n}\right)-1\right\}+\frac{1}{\sqrt{n}}\left\{\frac{1}{2} \hat{k}_{11}+\frac{1}{6} \hat{k}_{12}\right\},
\end{aligned}
$$

w.here $K_{n}=L_{1}$ and $\hat{\xi}=-\hat{k}_{12} /(3 \sqrt{n})$. Note that Sun et al. [10] defined $K_{n}=-L_{1}$, whereas our simulation results showed that it should be $K_{n}=L_{1}$. A major drawback of the two corrected tests is that they are more complicated test statistics involving higher-order moment estimations, which makes it difficult to derive their distributions under the alternative. Thus, they cannot be used for the study design.
Since $n^{-1} \rightarrow E_{H_{0}}(\Lambda(X))$ and $n^{-1} O \rightarrow E_{H_{0}}(\Delta)$, and $E_{H_{0}}(\Lambda(X))=E_{H_{0}}(\Delta)=\operatorname{Var}_{H_{0}}(W)$ as shown in the Appendix, thus, to correct the conservativeness of the original one-sample log-rank test $L_{1}$, we propose a new one-sample logrank test which is defined as

$$
L_{4}=\frac{O-E}{\sqrt{(O+E) / 2}} .
$$

In counting-process formulation, it is given by

$$
L_{4}=W / \hat{v}
$$

where

$$
W=n^{-1 / 2} \sum_{i=1}^{n} \int_{0}^{\infty}\left\{d N_{i}(x)-Y_{i}(x) d \Lambda_{0}(x)\right\}
$$

and

$$
\hat{v}^{2}=n^{-1 / 2} \sum_{i=1}^{n} \int_{0}^{\infty}\left\{d N_{i}(x)+Y_{i}(x) d \Lambda_{0}(x)\right\} / 2 .
$$

As shown in the Appendix, under the null hypothesis,

$$
\hat{v}^{2} \rightarrow v^{2}=\left\{E_{H_{0}}(\Delta)+E_{H_{0}}(\Lambda(X))\right\} / 2=\operatorname{Var}_{H_{0}}(W) .
$$

Therefore, again by counting-process central limit theorem under the null hypothesis, $L_{4}$ is asymptotically standard normal distribution. Hence, we reject the null hypothesis $H_{0}$ if $L_{4}=W / \hat{v}<-z_{1-\alpha}$.

Simulation studies are conducted in Section 4 to compare the empirical type I error and power of the original one-sample log-rank test $L_{1}$ to that of the two corrections $L_{2}$ and $L_{3}$, and the new test $L_{4}$.

\section{Sample Size Calculation}

To design the study, sample size must be calculated to detect a specified survival difference at the alternative $\Lambda(t)=\Lambda_{1}(t)\left(<\Lambda_{0}(t)\right)$, given the type I error $\alpha$ and power $1-\beta$. For the sample size calculation, the exact variance of $W$ has been derived by $\mathrm{Wu}$ [11]. Let the exact mean and variance of $\mathrm{W}$ at the alternative be $E_{H_{1}}(W)=\sqrt{n} \omega$ and $\operatorname{Var}_{H_{1}}(W)=\sigma^{2}$, respectively, where $\omega$ and $\sigma^{2}$ are given in the Appendix. By central limit theorem, $(W-\sqrt{n} \omega) / \sigma$ is approximately standard normal distribution under $H_{1}$. Under the alternative hypothesis,

$$
\hat{\sigma}^{2}=n^{-1} \sum_{i=1}^{n} \int_{0}^{\infty} Y_{i}(x) d \Lambda_{0}(x) \rightarrow \sigma_{0}^{2}=\int_{0}^{\infty} G(x) S_{1}(x) d \Lambda_{0}(x),
$$

and the power of the one-sample log-rank test $L_{1}=W / \hat{\sigma}$ should satisfy the following equations:

$$
\begin{aligned}
1-\beta=P\left(L_{1}<-z_{1-\alpha}\right) & \simeq P\left(\frac{W-\sqrt{n} \omega}{\sigma}<-\frac{\sigma_{0}}{\sigma} z_{1-\alpha}-\frac{\sqrt{n} \omega}{\sigma} \mid H_{1}\right) \\
& \simeq \Phi\left(\frac{\sigma_{0}}{\sigma} z_{1-\alpha}-\frac{\sqrt{n} \omega}{\sigma}\right) .
\end{aligned}
$$
by

Therefore, the required sample size for the test statistic $L_{1}$ is given

$$
n=\frac{\left(\sigma_{0} z_{1-\alpha}+\sigma z_{1-\beta}\right)^{2}}{\omega^{2}},
$$

where $\omega=\sigma_{1}^{2}-\sigma_{0}^{2}$ and $\sigma^{2}=p_{1}-p_{1}^{2}+2 p_{00}-p_{0}^{2}-2 p_{01}+2 p_{0} p_{1}$, with $\sigma_{0}^{2}, \sigma_{1}^{2}, p_{0} p_{1}, p_{00}$ and $p_{01}$ given in the Appendix.

Similarly, under the alternative, $\hat{v}^{2} \rightarrow \bar{\sigma}^{2}=\left(\sigma_{1}^{2}+\sigma_{0}^{2}\right) / 2$ (see Appendix); thus, the power of the new one-sample log-rank test $L_{4}=W / \hat{v}$ should satisfy the following equations: 


$$
\begin{aligned}
1-\beta=P\left(L_{4}<-z_{1-\alpha}\right) & \simeq P\left(\frac{W-\sqrt{n} \omega}{\sigma}<-\frac{\bar{\sigma}}{\sigma} z_{1-\alpha}-\frac{\sqrt{n} \omega}{\sigma} \mid H_{1}\right) \\
& \simeq \Phi\left(\frac{\bar{\sigma}}{\sigma} z_{1-\alpha}-\frac{\sqrt{n} \omega}{\sigma}\right) .
\end{aligned}
$$

Therefore, the required sample size for test statistic $L_{4}$ is given by

$$
n=\frac{\left(\bar{\sigma} z_{1-\alpha}+\bar{\sigma} z_{1-\beta}\right)^{2}}{\omega^{2}}
$$

where $\bar{\sigma}^{2}, \sigma^{2}$, and $\omega$ are the same as given above.

\section{Simulation Studies}

To study the performance of the two one-sample log-rank tests and their sample size formulas, we conducted simulation studies to compare the empirical power and type I error under different scenarios. In simulation studies, the survival distribution of the standard population was taken as the Weibull distribution $S_{0}(x)=e^{-\log (2)\left(x / m_{0}\right)^{k}}$, or cumulative hazard function $\Lambda_{0}(x)=\log (2)\left(x / m_{0}\right)^{\kappa}$, with a known shape parameter $\kappa$ and median survival time $\mathrm{m} 0$ under the null. Assume that the cumulative hazard function at the alternative is $\Lambda_{1}(x)=\log (2)\left(x / m_{1}\right)^{\kappa}$ with a common shape parameter $\kappa$, where the median survival time under the alternative $m_{1}>m_{0}$. Therefore, the underlying Weibull model is a proportional hazards model with hazard ratio $\delta=\left(m_{1} / m_{0}\right)^{\kappa}$. The parameter settings for the simulation studies were set to $\kappa=0.1,0.25,1,2$, and 5 to reflect cases of decreasing $(\kappa<1)$, constant $(\kappa=1)$ and increasing $(\kappa>1)$ hazard functions. The hazard ratio $\delta$ under the alternative hypothesis was set to $1.2-2.0$, with other parameters fixed as follows: $m_{0}=1$, accrual period $t_{a}=3$, and follow-up time $t_{f}=1$.

We assumed that subjects were recruited with a uniform distribution over the accrual period $t_{a}$ and followed for $t_{f}$. We further assumed that no subject was lost to follow-up or drop-out during the study. Then the censoring time is uniformly distributed on the interval $\left[t_{\rho} t_{a}+t_{f}\right]$. Thus, under the Weibull model, quantities $p_{0}, p_{1}, p_{00}$, and $p_{01}$, hence $\sigma_{0}^{2}, \sigma_{1}^{2}, \omega, \sigma^{2}$ can be calculated by numerical integrations. Given the nominal significance level of 0.05 and power of $90 \%$, the required sample sizes for each design scenario were calculated for test statistics $L_{1}$ and $L_{4}$ (Table 1). The empirical type I error and power for the corresponding design were also simulated based on 100,000 samples generated from the Weibull distribution (Table 1). To compare the four test statistics, we also simulated the empirical type I error and power of the four test statistics $L_{1}-L_{4}$ given the same sample size $n=30$, 50, 100, and 200 (Table 2).

The sample size calculation (Table 1) showed that the original onesample log-rank test $L_{1}$ required a larger sample size than that of the new test $L_{4}$. The simulated empirical type I errors for the corresponding sample size showed that the type I error of $L_{1}$ was always less than the nominal level. Thus, the original one-sample log-rank test $L_{1}$ was conservative. The empirical type I errors of the new test $L_{4}$ were close to the nominal level in most scenarios and were slightly liberal when the sample size was small. The simulation results in Table 2 with the same sample size further confirmed that the test $L_{1}$ was conservative and that $L_{4}$ preserved the type I error well and had a higher power than that of the $L_{1}$. It is consistent with the results from sample size calculations that $L_{4}$ had a smaller sample size than $\operatorname{did} L_{1}$. Simulations were also done for

\begin{tabular}{|c|c|c|c|c|c|c|c|c|c|c|}
\hline \multirow[b]{2}{*}{$\kappa$} & \multirow[b]{2}{*}{ Test } & \multicolumn{3}{|c|}{$\delta=1.2$} & \multicolumn{3}{|c|}{$\delta=1.3$} & \multicolumn{3}{|c|}{$\delta=1.4$} \\
\hline & & $n$ & $\alpha$ & $1-\beta$ & $\mathrm{n}$ & $\alpha$ & $1-\beta$ & $\mathrm{n}$ & $\alpha$ & $1-\beta$ \\
\hline \multirow[t]{2}{*}{0.1} & $\mathrm{~L}_{1}$ & 534 & .048 & .903 & 269 & .046 & .906 & 169 & .044 & .907 \\
\hline & $\mathrm{L}_{4}$ & 508 & .051 & .897 & 250 & .051 & .896 & 155 & .053 & .893 \\
\hline \multirow[t]{2}{*}{0.5} & $\mathrm{~L}_{1}$ & 432 & .047 & .905 & 217 & .046 & .907 & 137 & .046 & .909 \\
\hline & $\mathrm{L}_{4}$ & 411 & .051 & .899 & 203 & .052 & .901 & 125 & .053 & .897 \\
\hline \multirow[t]{2}{*}{1.0} & $\mathrm{~L}_{1}$ & 356 & .047 & .907 & 178 & .045 & .909 & 112 & .044 & .912 \\
\hline & $\mathrm{L}_{4}$ & 339 & .050 & .904 & 167 & .050 & .903 & 103 & .049 & .905 \\
\hline \multirow[t]{2}{*}{2.0} & $\mathrm{~L}_{1}$ & 306 & .046 & .910 & 153 & .043 & .915 & 97 & .042 & .922 \\
\hline & $\mathrm{L}_{4}$ & 292 & .049 & .907 & 144 & .049 & .910 & 89 & .048 & .913 \\
\hline \multirow[t]{3}{*}{5.0} & $\mathrm{~L}_{1}$ & 288 & .046 & .912 & 144 & .044 & .917 & 91 & .042 & .925 \\
\hline & L4 & 275 & .050 & .909 & 135 & .049 & .912 & 84 & .049 & .916 \\
\hline & & \multicolumn{3}{|c|}{$\delta=1.5$} & \multicolumn{3}{|c|}{$\delta=1.6$} & \multicolumn{3}{|c|}{$\delta=1.7$} \\
\hline$\kappa$ & Test & $n$ & $\alpha$ & $1-\beta$ & $n$ & $\alpha$ & $1-\beta$ & $\mathrm{n}$ & $\alpha$ & $1-\beta$ \\
\hline \multirow[t]{2}{*}{0.1} & $\mathrm{~L}_{1}$ & 121 & .045 & .908 & 93 & .044 & .909 & 75 & .043 & .911 \\
\hline & $\mathrm{L}_{4}$ & 109 & .053 & .897 & 82 & .052 & .893 & 66 & .052 & .894 \\
\hline \multirow[t]{2}{*}{0.5} & $\mathrm{~L}_{1}$ & 97 & .044 & .912 & 75 & .042 & .913 & 60 & .043 & .910 \\
\hline & $\mathrm{L}_{4}$ & 88 & .053 & .900 & 66 & .053 & .898 & 53 & .053 & .900 \\
\hline \multirow[t]{2}{*}{1.0} & $\mathrm{~L}_{1}$ & 80 & .043 & .916 & 61 & .042 & .916 & 49 & .041 & .919 \\
\hline & $\mathrm{L}_{4}$ & 72 & .051 & .904 & 55 & .050 & .907 & 44 & .051 & .908 \\
\hline \multirow[t]{2}{*}{2.0} & $\mathrm{~L}_{1}$ & 69 & .042 & .927 & 53 & .040 & .929 & 43 & .040 & .934 \\
\hline & $\mathrm{L}_{4}$ & 63 & .049 & .918 & 47 & .050 & .916 & 38 & .049 & .921 \\
\hline \multirow[t]{4}{*}{5.0} & $\mathrm{~L}_{1}$ & 65 & .040 & .930 & 50 & .039 & .935 & 40 & .040 & .937 \\
\hline & $\mathrm{L}_{4}$ & 59 & .049 & .919 & 45 & .049 & .924 & 36 & .048 & .928 \\
\hline & & \multicolumn{3}{|c|}{$\delta=1.8$} & \multicolumn{3}{|c|}{$\delta=1.9$} & \multicolumn{3}{|c|}{$\delta=2.0$} \\
\hline & Test & $n$ & $\alpha$ & $1-\beta$ & $n$ & $\alpha$ & $1-\beta$ & $n$ & $\alpha$ & $1-\beta$ \\
\hline \multirow[t]{2}{*}{0.1} & $\mathrm{~L}_{1}$ & 63 & .041 & .911 & 54 & .042 & .911 & 47 & .041 & .909 \\
\hline & $\mathrm{L}_{4}$ & 54 & .055 & .893 & 46 & .056 & .891 & 40 & .055 & .892 \\
\hline \multirow[t]{2}{*}{0.5} & $\mathrm{~L}_{1}$ & 50 & .041 & .912 & 43 & .041 & .913 & 38 & .041 & .915 \\
\hline & $\mathrm{L}_{4}$ & 44 & .055 & .902 & 37 & .053 & 897 & 32 & .054 & .894 \\
\hline \multirow[t]{2}{*}{1.0} & $\mathrm{~L}_{1}$ & 41 & .040 & .921 & 35 & .040 & .921 & 31 & .040 & .925 \\
\hline & $\mathrm{L}_{4}$ & 36 & .051 & .908 & 31 & .052 & .911 & 27 & .052 & .912 \\
\hline \multirow[t]{2}{*}{2.0} & $\mathrm{~L}_{1}$ & 36 & .038 & .938 & 31 & .038 & .940 & 27 & .038 & .942 \\
\hline & $\mathrm{L}_{4}$ & 31 & .048 & .920 & 27 & .050 & .925 & 23 & .049 & .922 \\
\hline \multirow[t]{2}{*}{5.0} & $\mathrm{~L}_{1}$ & 34 & .040 & .943 & 29 & .038 & .945 & 25 & .036 & .943 \\
\hline & $\mathrm{L}_{4}$ & 30 & .048 & .930 & 25 & .048 & .929 & 22 & .048 & .932 \\
\hline
\end{tabular}
the two corrected tests $L_{2}$ and $L_{3}$. The results showed that $L_{2}$ preserved the type I error well and had a higher power than $L_{1}$ and $L_{2}$, and $L_{3}$
Table 1: Sample size, simulated empirical type I error $(\alpha)$, and power $(1-\beta)$ of test statistics $L_{1}$ and $L_{4}$ based on 100,000 simulation runs from the Weibull distribution with nominal type I error of 0.05 and power of $90 \%$ (one-sided test).

was slightly conservative when sample size was small. Furthermore, the empirical type I error and power of test $L_{4}$ were also comparable to the two corrections $L_{2}$ and $L_{3}$.

To compare the null distribution functions of the four test statistics to the standard normal for small sample sizes, we conducted 100,000 simulation runs to simulate the empirical distribution functions of $L_{1}-$ $L_{4}$ under the null with sample size $n=30$ to 200 (Table 3). The simulation results showed that the distribution of $L_{1}$ had a light left tail, while $L_{4}$ had a slightly heavier left tail than a standard normal distribution function. The results explained the observations from previous simulations that the test $L_{1}$ was conservative and $L_{4}$ was slightly liberal when the sample size was small. The distribution of $L_{2}$ was almost the same as the standard normal distribution function, and the distribution of $L_{3}$ had a slightly lighter left tail when sample size was small. Overall, $L_{4}$ preserved type I error well and had power higher than that of $L_{1}-L_{3}$. The distribution function of $L_{4}$ was also close to the standard normal and comparable to that of $L_{2}$ and $L_{3}$. The major advantage of $L_{4}$ is its simplicity and ease with which it derives the asymptotic distribution under the alternative. Therefore, the proposed new one-sample logrank test $L_{4}$ is preferred for the study design and data analysis of a study comparing the survival of a sample to that of the standard population. 


\begin{tabular}{|c|c|c|c|c|c|c|c|c|c|c|c|c|}
\hline \multicolumn{13}{|c|}{$\delta$} \\
\hline$\kappa$ & $\mathrm{n}$ & Test & 1.0 & 1.2 & 1.3 & 1.4 & 1.5 & 1.6 & 1.7 & 1.8 & 1.9 & 2.0 \\
\hline \multirow[t]{16}{*}{0.5} & 30 & & .040 & .169 & .264 & .369 & .479 & .577 & .665 & .737 & .799 & .846 \\
\hline & & $\mathrm{L}_{2}$ & .049 & .197 & .299 & .411 & .523 & .622 & .704 & .774 & .829 & .870 \\
\hline & & $\mathrm{L}_{3}$ & .046 & .190 & .290 & .400 & .512 & .612 & .695 & .765 & .821 & 864 \\
\hline & & $\mathrm{L}_{4}$ & .055 & .210 & .317 & .430 & .539 & .636 & .719 & .783 & .839 & .879 \\
\hline & 50 & $\mathrm{~L}_{1}$ & .042 & .241 & .388 & .544 & .677 & .784 & .863 & .912 & .945 & .968 \\
\hline & & $\mathrm{L}_{2}$ & .051 & .267 & .422 & .575 & .708 & .807 & .878 & .926 & .955 & .973 \\
\hline & & $\mathrm{L}_{3}$ & .049 & .260 & .414 & .567 & .701 & .801 & .874 & .923 & .953 & .972 \\
\hline & & $\mathrm{L}_{4}$ & .054 & 279 & . 435 & 591 & .718 & 817 & 887 & .930 & .957 & .975 \\
\hline & 100 & $\mathrm{~L}_{1}$ & .043 & .399 & .635 & .812 & .919 & .967 & .988 & 996 & .999 & 1 \\
\hline & & $\mathrm{L}_{2}$ & .050 & .420 & .656 & .831 & .926 & .972 & .990 & .996 & .999 & 1 \\
\hline & & $\mathrm{L}_{3}$ & .048 & .414 & .651 & .827 & .924 & .971 & .989 & .996 & .999 & 1 \\
\hline & & $\mathrm{L}_{4}$ & .051 & .431 & .665 & .833 & .930 & .973 & .991 & .997 & .999 & 1 \\
\hline & 200 & $\mathrm{~L}_{1}$ & .046 & .635 & .885 & .976 & .996 & 1 & 1 & 1 & 1 & 1 \\
\hline & & $\mathrm{L}_{2}$ & .050 & .651 & .893 & .979 & .997 & 1 & 1 & 1 & 1 & 1 \\
\hline & & $\mathrm{L}_{3}$ & .049 & .647 & .891 & .978 & .996 & 1 & 1 & 1 & 1 & 1 \\
\hline & & $\mathrm{L}_{4}$ & .051 & .656 & .896 & .979 & .997 & 1 & 1 & 1 & 1 & 1 \\
\hline \multirow[t]{16}{*}{1} & 30 & $\mathrm{~L}_{1}$ & .039 & .193 & .316 & .441 & .569 & .673 & .760 & .827 & .879 & .916 \\
\hline & & $\mathrm{L}_{2}$ & .049 & .226 & .356 & .487 & .609 & .715 & .796 & .856 & .900 & .932 \\
\hline & & $\mathrm{L}_{3}$ & .043 & .207 & .331 & .461 & .583 & .693 & .778 & .841 & .889 & .924 \\
\hline & & $\mathrm{L}_{4}$ & .051 & .232 & .365 & .492 & .619 & .718 & .797 & .858 & .903 & 933 \\
\hline & 50 & $\mathrm{~L}_{1}$ & .041 & .281 & .460 & .631 & .768 & .861 & .924 & .959 & .979 & 988 \\
\hline & & $\mathrm{L}_{2}$ & .050 & 308 & .493 & .663 & .794 & .879 & .935 & .966 & .982 & .991 \\
\hline & & $\mathrm{L}_{3}$ & .045 & .291 & .473 & .644 & .780 & .869 & .929 & .962 & .980 & .990 \\
\hline & & $\mathrm{L}_{4}$ & .051 & .317 & .501 & .669 & .797 & .882 & .938 & .967 & .983 & .991 \\
\hline & 100 & $\mathrm{~L}_{1}$ & .044 & .461 & .718 & .884 & .959 & .988 & .997 & .999 & 1 & 1 \\
\hline & & $\mathrm{L}_{2}$ & .051 & .487 & .738 & .894 & .964 & .990 & .997 & .999 & 1 & 1 \\
\hline & & $\mathrm{L}_{3}$ & .047 & .473 & .726 & .887 & .962 & .989 & .997 & .999 & 1 & 1 \\
\hline & & $\mathrm{L}_{4}$ & .052 & .490 & .741 & .897 & .965 & .990 & .997 & .999 & 1 & 1 \\
\hline & 200 & $\mathrm{~L}_{1}$ & .046 & .716 & .935 & .992 & .999 & & & 1 & 1 & 1 \\
\hline & & $\mathrm{L}_{2}$ & .051 & .732 & .941 & .992 & .999 & 1 & 1 & 1 & 1 & 1 \\
\hline & & $\mathrm{L}_{3}$ & .048 & .725 & .939 & .992 & .999 & 1 & 1 & 1 & 1 & 1 \\
\hline & & $\mathrm{L}_{4}$ & .051 & .734 & .942 & .993 & .999 & 1 & 1 & 1 & 1 & 1 \\
\hline \multirow[t]{16}{*}{2} & 30 & $\mathrm{~L}_{1}$ & .037 & .220 & .363 & .514 & .647 & .758 & .836 & .894 & .933 & .959 \\
\hline & & $\mathrm{L}_{2}$ & .051 & .262 & .413 & .560 & .694 & .792 & .867 & .916 & .948 & 967 \\
\hline & & $\mathrm{L}_{3}$ & .040 & .225 & .369 & .516 & .652 & .760 & .843 & .898 & .936 & .950 \\
\hline & & $\mathrm{L}_{4}$ & .048 & .256 & .407 & .557 & .687 & .791 & .862 & .911 & .945 & .967 \\
\hline & 50 & $\mathrm{~L}_{1}$ & .041 & .317 & .526 & .709 & .838 & .916 & .961 & .982 & .992 & .997 \\
\hline & & $\mathrm{L}_{2}$ & .050 & .354 & .564 & .739 & .859 & .931 & .969 & .986 & .994 & 998 \\
\hline & & $\mathrm{L}_{3}$ & .041 & .322 & .530 & .711 & .839 & .919 & .963 & .982 & .992 & 997 \\
\hline & & $\mathrm{L}_{4}$ & .050 & .349 & .561 & .738 & .858 & .928 & .968 & .985 & .993 & .997 \\
\hline & 100 & $\mathrm{~L}_{1}$ & .042 & .519 & .789 & .929 & .981 & .996 & .999 & 1 & 1 & 1 \\
\hline & & $\mathrm{L}_{2}$ & .051 & .551 & .807 & .937 & .984 & .996 & .999 & 1 & 1 & 1 \\
\hline & & $\mathrm{L}_{3}$ & .045 & .527 & .791 & .930 & .981 & .996 & .999 & 1 & 1 & 1 \\
\hline & & $\mathrm{L}_{4}$ & .049 & .546 & .807 & .937 & .983 & .996 & .999 & 1 & 1 & 1 \\
\hline & 200 & $\mathrm{~L}_{1}$ & .044 & .781 & \multicolumn{2}{|c|}{$.966^{19} .997$} & 1 & 1 & 1 & 1 & 1 & 1 \\
\hline & & $\mathrm{L}_{2}$ & .050 & .796 & .968 & .997 & 1 & 1 & 1 & 1 & 1 & 1 \\
\hline & & $\mathrm{L}_{3}$ & .046 & .784 & .965 & .997 & 1 & 1 & 1 & 1 & 1 & 1 \\
\hline & & $\mathrm{L}_{4}$ & .049 & .795 & .969 & .998 & 1 & 1 & 1 & 1 & 1 & 1 \\
\hline
\end{tabular}

Table 2: Simulation studies for empirical type I error $(\delta=1)$ and power $(\delta>1)$ of four test statistics, $L_{1}-L_{4}$, based on 100,000 simulation runs from the Weibull distribution with nominal type I error of 0.05 (one-sided test).

\section{An Example}

This example, Example V.1.5, is taken from Anderson et al. [4]. During the period 1962-1977, 205 patients with malignant melanoma had a radical operation performed at the Department of Plastic Surgery, University Hospital of Odense, Demark. A total of 57 patients died of malignant melanoma, 14 died of other causes; and the remaining 134 patients were alive as of January 1, 1978. If one is interested in studying deaths due to causes other than malignant melanoma and comparing those data to the standard life tables for the Danish population during 1971-1975, then using classical one-sample log-rank test, there are $O=14$ observed deaths versus $E=21.244$ expected deaths (see Anderson et al., page 338), yielding an observed value of the test statistic $L_{1}=(O-E) / \sqrt{E}=-1.57$, which is not significant compared to $-z_{1-\alpha}=-1.645$ for the significance level $\alpha=0.05$. However, the new

\begin{tabular}{|c|c|c|c|c|c|c|c|c|c|}
\hline \multicolumn{10}{|c|}{$x$} \\
\hline$\kappa$ & $\mathrm{n}$ & Test & -3.0 & -1.96 & -0.67 & 0.0 & 0.67 & 1.96 & 3.0 \\
\hline \multirow[t]{16}{*}{0.5} & 30 & $\mathrm{~L}_{1}$ & .0003 & .0169. & .2428. & .4949. & .7352 & 9632 & 9959 \\
\hline & & $\mathrm{L}_{2}$ & .0013 & .0242 & .2539 & . 49887 & .7442 & .9767 & .9991 \\
\hline & & L3 & .0012 & .0228 & .2450 & .4888 & .7368 & .9748 & .9989 \\
\hline & & $\mathrm{L}_{4}$ & .0021. & .0285 & .2504 & .4949. & .7440 & 9783 & .9993 \\
\hline & 50 & $\mathrm{~L}_{1}$ & .0006 & .0190 & .2446 & .4958 & .7412 & .9669 & .9964 \\
\hline & & $\mathrm{L}_{2}$ & .0013 & .0251 & .2524 & .4997 & .7498 & .9753 & .9991 \\
\hline & & $\mathrm{L}_{3}$ & .0012 & .0240 & .2461 & .4920 & .7437 & .9742 & .9989 \\
\hline & & $\mathrm{L}_{4}$ & .0021 & .0283 & .2506 & .4958 & .7477 & .9771 & .9991 \\
\hline & 100 & $L_{1}$ & .0008 & .0210 & .2470 & .4974 & .7430 & .9692 & .9977 \\
\hline & & $\mathrm{L}_{2}$ & .0012 & .0254 & .2527 & .4995 & .7481 & .9756 & .9989 \\
\hline & & $\mathrm{L}_{3}$ & .0011 & .0245 & .2479 & .4942 & .7438 & .9748 & .9988 \\
\hline & & $\mathrm{L}_{4}$ & .0019 & .0280 & .2512 & .4974 & .7475 & .9770 & .9989 \\
\hline & 200 & $\mathrm{~L}_{1}$ & .0008 & .0210 & .2480 & .4969 & .7447 & .9702 & .9978 \\
\hline & & $\mathrm{L}_{2}$ & .0012 & .0252 & .2527 & .4999 & .7492 & .9754 & .9988 \\
\hline & & $\mathrm{L}_{3}$ & .0012 & .0246 & .2491 & .4960 & .7461 & .9748 & .9987 \\
\hline & & $\mathrm{L}_{4}$ & .0016 & .0259 & .2512 & .4969 & .7479 & .9758 & .9988 \\
\hline \multirow[t]{16}{*}{1} & 30 & $\mathrm{~L}_{1}$ & .0005 & 0167 & .2374 & .4870 & .7334 & .9628 & .9961 \\
\hline & & $\mathrm{L}_{2}$ & .0011 & .0248 & .2517 & .4999 & .7464 & .9756 & .9992 \\
\hline & & L3 & .0009 & 0210 & 2319 & .4750 & .7291 & .9724 & .9989. \\
\hline & & $\mathrm{L}_{4}$ & .0019 & .0266 & .2440 & .4870 & .7412 & .9771 & .9994 \\
\hline & 50 & $\mathrm{~L}_{1}$ & .0005 & .0192 & .2427 & .4908 & .7367 & .9668 & .9969 \\
\hline & & $\mathrm{L}_{2}$ & .0012 & .0251 & .2532 & .5001 & .7458 & .9754 & .9989 \\
\hline & & $\mathrm{L}_{3}$ & .0010 & .0221 & .2382 & .4814 & .7316 & .9728 & .9988 \\
\hline & & $\mathrm{L}_{4}$ & .0018 & .0271 & .2480 & .4908 & .7430 & .9770 & .9991 \\
\hline & 100 & $\mathrm{~L}_{1}$ & .0008 & .0199 & .2460 & .4956 & .7415 & .9695 & .9977 \\
\hline & & $\mathrm{L}_{2}$ & .0013 & .0250 & .2514 & .4995 & .7466 & .9748 & .9988 \\
\hline & & $\mathrm{L}_{3}$ & .0011 & .0232 & .2404 & .4865 & .7368 & .9731 & .9986 \\
\hline & & $\mathrm{L}_{4}$ & .0020 & .0256 & .2499 & .4956 & .7456 & .9767 & .9990 \\
\hline & 200 & $\mathrm{~L}_{1}$ & .0009 & .0214 & .2484 & .4958 & .7423 & .9712 & .9979 \\
\hline & & $\mathrm{L}_{2}$ & .0013 & .0246 & .2526 & .5008 & .7483 & .9748 & .9984 \\
\hline & & $\mathrm{L}_{3}$ & .0012 & .0233 & .2451 & .4916 & .7410 & .9736 & .9982 \\
\hline & & $\mathrm{L}_{4}$ & .0016 & .0251 & .2513 & .4958 & .7453 & .9760 & .9988 \\
\hline \multirow[t]{17}{*}{2} & 30 & $\mathrm{~L}_{1}$ & .0005 & .0167 & .2308 & .4789 & .7256 & .9626 & .9960 \\
\hline & & $\mathrm{L}_{2}$ & .0014 & .0262 & .2532 & .5007 & .7451 & .9763 & .9990 \\
\hline & & $\mathrm{L}_{3}$ & .0007 & .0194 & .2201 & .4630 & .7179 & .9718 & .9986 \\
\hline & & $\mathrm{L}_{4}$ & .0016 & .0255 & .2373 & .4789 & .7329 & .9765 & .9992 \\
\hline & 50 & $\mathrm{~L}_{1}$ & .0006 & .0180 & .2344 & .4834 & .7297 & .9656 & .9970 \\
\hline & & $\mathrm{L}_{2}$ & .0012 & .0252 & .2528 & .4994 & .7461 & .9742 & .9987 \\
\hline & & $\mathrm{L}_{3}$ & .0010 & .0201 & .2273 & .4689 & .7236 & .9704 & .9984 \\
\hline & & $\mathrm{L}_{4}$ & .0016 & .0250 & .2395 & .4834 & .7351 & .9757 & .9991 \\
\hline & 100 & $\mathrm{~L}_{1}$ & .0008 & .0192 & .2398 & .4899 & .7374 & .9694 & .9977 \\
\hline & & $\mathrm{L}_{2}$ & .0012 & .0245 & .2512 & .4980 & .7481 & .9749 & .9988 \\
\hline & & $\mathrm{L}_{3}$ & .0009 & .0211 & .2331 & .4760 & .7307 & .9718 & .9986 \\
\hline & & $\mathrm{L}_{4}$ & .0016 & .0247 & .2437 & .4899 & .7415 & .9762 & .9990 \\
\hline & 200 & $\mathrm{~L}_{1}$ & .0008 & .0206 & .2445 & .4947 & .7415 & .9713 & .9979 \\
\hline & & $\mathrm{L}_{2}$ & .0014 & .0251 & .2501 & .4992 & .7472 & .9743 & .9987 \\
\hline & & $\mathrm{L}_{3}$ & .0012 & .0225 & 371 & .4838 & .7351 & .9722 & .9985 \\
\hline & & $\mathrm{L}_{4}$ & .0014 & .0244 & .2470 & .4947 & .7444 & .9759 & .9988 \\
\hline & & $\Phi(x)$ & .0013 & .0250 & .2514 & .5000 & .7486 & .9750 & .9987 \\
\hline
\end{tabular}

Table 3: Simulated distribution functions of $\mathrm{L}_{1}-\mathrm{L}_{4}$ compared to the standard normal distribution function based on 100,000 simulation runs from the Weibull distribution. 
one-sample log-rank test $L_{4}=(O-E) / \sqrt{O+E / 2}=-1.726<-1.645$, or a $p$-value of 0.042 ; thus, we can claim that the mortality from other causes among patients with melanoma is significantly lower than that of the Danish general population.

\section{Conclusions}

A simple one-sample log-rank test is proposed, and its sample size formula is derived. Simulation results showed that the new test $L_{4}$ preserves the type I error well and is comparable to the two corrections based on Edgeworth expansion [10]. The proposed new test $L_{4}$ had power higher than that of the original test $L_{1}$ and the two corrections $L_{2}$ and $L_{3}$. The sample size formula derived from the new test statistic $L_{4}$ provides adequate power for the study design. To use the one-sample log-rank test to design a study and make inferences, the underlying distribution or hazard function of the standard population has to be correctly specified, b ecause b oth s tudy design a nd inference d epend on the validity of this assumption. In an epidemiologic study, the standard population is often well defined. Therefore, one can use the method proposed by Finkelstein et al. [1] to calculate the expected number of events and estimate the survival distribution of the standard population. In a phase II clinical trial, the survival function of the historical control can be estimated from meta-analysis or other sources [10]. Nevertheless, a simple one-sample log-rank test is proposed, and its sample size formula is derived to provide a study design that preserves the type I error and ensures sufficient power to detect the difference of survival distributions between a sample and a standard population.

\section{Acknowledgment}

This work was supported in part by the National Cancer Institute $(\mathrm{NCl})$ support grant P30CA021765-35

\section{References}

1. Finkelstein DM, Muzikansky A, Schoenfeld DA (2003) Comparing survival of a sample to that of a standard population. J Natl Cancer Inst 95: 1434-1439.

2. Breslow NE (1975) Analysis of survival data under the proportional hazards model. International statistical Review 43: 44-58.

3. Hyde J (1977) Testing survival under right censoring and left truncation Biometrika 64: 225-230.

4. Anderson PK, Borgan O, Gill RD, Keiding N (1993) Statistical models based on counting processes, Springer, New York.

5. Gill MH, Ware JH (1979) Comparing observed life table data with a known survival curve in the presence of random censorship. Biometrics 35: 385-391.

6. Berry G (1983) The analysis of mortality by the subject-years method. See comment in PubMed Commons below Biometrics 39: 173-184.

7. Woolson RF (1981) Rank-tests and a one-sample log-rank test for comparing observed survival-data to a standard population. Biometrics 37: 687-696.

8. Kwak M, Jung SH (2014) Phase II clinical trials with time-to-event endpoints: optimal two-stage designs with one-sample log-rank test. Stat Med 33: 2004 2016.

9. Jung SH (2013) Randomized phase II cancer clinical trial. CRC Press: Chapman \& Hall.

10. Sun X, Peng P, Tu D (2011) Phase II cancer clinical trials with a one-sample log-rank test and its corrections based on the Edgeworth expansion. See comment in PubMed Commons below Contemp Clin Trials 32: 108-113.

11. Wu J (2014) Sample size calculation for the one-sample log-rank test Technique report, Department of Biostatistics, St. Jude Children's Research Hospital.

12. Fleming TR, Harrington DP (1991) Counting processes and survival analysis. John Wiley and Sons, New York. 